\title{
Two- Versus Four-Times Weekly Acupuncture-Like Transcutaneous Electrical Nerve Stimulation for Treatment of Radiation-Induced Xerostomia: A Pilot Study
}

\author{
Austin J. lovoli, BSc, Alexander Ostrowski, BSc, ${ }^{2}$ Charlotte I. Rivers, $\mathrm{MD}^{3}$ \\ Gregory M. Hermann, MD, MPH, Adrienne Groman, MS, \\ Austin Miller, $\mathrm{PhD}^{4}$ and Anurag K. Singh, $\mathrm{MD}^{3}$
}

\begin{abstract}
Background: Xerostomia occurs in the majority of patients undergoing chemoradiation therapy for head and neck cancer (HNC). Acupuncture-like transcutaneous electrical nerve stimulation (ALTENS) treatment has been studied as an encouraging modality to improve salivary function and related symptoms. The purpose of this study was to compare ALTENS treatment by using a four-times weekly schedule for 6 weeks versus a twice-weekly schedule for 12 weeks with a validated xerostomia scale at 15 months from the start of ALTENS treatment.

Materials and Methods: This single-center randomized study was conducted in 30 patients treated with radiotherapy with or without chemotherapy for HNC between 2014 and 2017, who had at least grade 1 or 2 symptomatic dry mouth (xerostomia) according to CTEP NCI Common Terminology Criteria for Adverse Events (CTCAE version 4.0). These patients were randomly assigned to receive ALTENS four-times weekly for 6 weeks or two-times weekly for 12 weeks. The University of Michigan 15-item Xerostomia-related Quality of Life Scale (XeQoLS) was administered at 6, 9, 15, and 21 months from the start of ALTENS treatment. A random-effects generalized linear model was used to model the overall XeQoLS score at the 15-month endpoint; adjusted for a random time effect, a fixed treatment arm, and interaction of time and treatment. Comparison between arms was based on a 0.05 nominal significance level.

Results: XeQoLS decreased for all patients (although not statistically for each arm) from a mean of 22 and 21 at baseline (in the four times per week and twice weekly arms) to 12 in both arms at 15 months, with no difference between arms $(p=0.68)$. There were no attributable grade 1-3 adverse events. Arms were balanced for age, gender, race, and baseline xerostomia.

Conclusions: This study demonstrates that both ALTENS regimens are safe, well tolerated, and appear to be equally effective. We now routinely make ALTENS units available for home use.
\end{abstract}

Keywords: ALTENS, xerostomia, head and neck cancer, QOL

\footnotetext{
${ }^{1}$ Jacobs School of Medicine and Biomedical Sciences, Buffalo, New York.

Departments of ${ }^{2}$ Information Technology, ${ }^{3}$ Radiation Medicine, and ${ }^{4}$ Biostatistics and Bioinformatics, Roswell Park Comprehensive Cancer Center, Buffalo, New York.
} 


\section{Introduction}

$\mathbf{X}$ EROSTOMIA OCCURS IN the majority of patients undergoing chemoradiation therapy (CRT) for head and neck cancer (HNC). Advances in radiation therapy, such as the development of intensity modulated radiation therapy (IMRT), allow sparing of the parotid glands from highdose radiation. ${ }^{1}$ Even low-dose radiation to the parotid glands, however, causes xerostomia in nearly all HNC patients to some extent. ${ }^{2}$ Individuals with xerostomia can develop problems with eating, speaking, swallowing, oral infections, and dental caries. ${ }^{3}$ Xerostomia is often permanent and contributes to decreased quality of life. Procholinergic salivary secretagogues such as pilocarpine are typically insufficient and are associated with significant adverse side effects. ${ }^{4}$ Randomized trials of pilocarpine in alleviating post-radiation xerostomia have been unable to demonstrate a beneficial effect when compared with placebo. ${ }^{5,6}$

Acupuncture-like transcutaneous electrical nerve stimulation (ALTENS) treatment has been studied as an encouraging modality to improve salivary function and related symptoms without the negative side effects of salivary secretagogues. ${ }^{7-10}$ ALTENS is performed by using a transcutaneous electrical nerve stimulation device routinely used for pain management that can simulate acupuncture without using needles. By utilizing appropriate acupuncture treatment points, single and multi-institutional studies have found reduction of radiation-induced xerostomia and improved quality of life with ALTENS therapy, given twice weekly for 12 weeks, 3-27 months after completing CRT. ${ }^{7-10}$ This twice weekly for 12 weeks ALTENS regimen was offered as a free adjuvant treatment to all of our patients with xerostomia; however, many patients requested a regimen with shorter overall duration that would be easier to schedule between other requirements such as travel for work or vacation. A few patients who were treated more frequently per week for fewer weeks reported excellent results.

Evidence suggests that ALTENS can treat radiationinduced xerostomia through stimulation of a neuroreflex involving the autonomic nervous system. ${ }^{11}$ Given this implication of neural stimulation as a mechanism of action, and our small retrospective experience, we hypothesized that increased frequency of ALTENS procedures may increase the neural action and, thus, improve the effectiveness of xerostomia reduction. In addition, to identify a shorter, potentially more convenient regimen, this singlecenter randomized study compared ALTENS twice weekly for 12 weeks versus four times weekly for 6 weeks.

\section{Materials and Methods}

We report results of a single-center, institutional review board approved randomized study (ClinicalTrials.gov identifier: NCT02474095). All patients provided written informed consent, and the study was conducted in accordance to all applicable local regulatory requirements and laws. Patients were randomized in a 1:1 ratio to either ALTENS twice weekly for 12 weeks or four times weekly for 6 weeks.

\section{Patient population}

Eligible patients were at least 18 years of age and had completed radiotherapy with or without chemotherapy for head and neck squamous cell carcinoma. The cisplatinbased chemotherapy (weekly or every 3 weeks) and IMRT regimens ( 70 Gy to the primary tumor and 56 Gy to the elective lymph nodes in 35 fractions) applied in this cohort have been previously described in detail. ${ }^{1,12}$ Patients were enrolled between November 2014 and July 2017. No patients had parotid glands surgically removed and every effort was made to limit the bilateral parotid dose. The standard institutional dose constraint to each parotid was $\mathrm{D} 50 \%<30 \mathrm{~Gy}$. Time from completion of radiotherapy to registration was at least 3 months and up to a maximum of 1 year.

All patients had: grade 1 or 2 symptomatic dry mouth (xerostomia) according to CTEP NCI Common Terminology Criteria for Adverse Events (CTCAE version 4.0), no clinical evidence of disease within 8 weeks of registration by imaging, and Eastern Cooperative Oncology Group (ECOG) performance status of $\leq 2$.

Patients were excluded from this study if: taking pilocarpine for ophthalmic or non-ophthalmic indications or medications that would induce xerostomia (tricyclic antidepressants, antihistamines with anticholinergic effects). Patients with a history of Sjogren's disease were also excluded.

\section{Acupuncture-like transcutaneous nerve stimulation treatment}

ALTENS was administered with a Codetron ${ }^{\mathrm{TM}}$ (model 902-C; EHM Rehabilitation Technologies Ltd., Ontario, Canada) TENS units and Karaya electrode pads as previously described in RTOG 0537. ${ }^{7}$ Bilateral acupuncture points SP6, ST36, and LI4 using uncommon electrodes as well as CV24 using a common electrode were stimulated. These acupuncture points were selected on the basis of the meridian principles described in Traditional Chinese Medicine and used in the phase I-II clinical trial by Wong et al. ${ }^{9}$ Sequences of $250 \mathrm{msec}$ square pulses with a $4 \mathrm{~Hz}$ repetition rate were delivered. Each acupuncture point, except CV24, was stimulated for $10 \mathrm{sec}$ at a time. CV24, the site for the common electrode, was stimulated throughout the treatment session. Stimulation intensity (between level 3 and 6 on the machine) was adjusted to produce a deep strong aching sensation at each acupuncture point. Random switching among electrodes enabled by the Codetron embedded random circuit was employed to prevent brain habituation to stimulation. All patients received 24 ALTENS sessions (20 min each), either 2 sessions per week for 12 weeks or 4 sessions per week over 6 weeks. Sessions were delivered at an academic institution within the Radiation Medicine Department by RTOG trained staff who were not blinded to the treatment arm.

\section{Quality of life and patient-reported xerostomia}

Study data were collected and managed by using a secure web application, Research Electronic Data Capture (REDCap), hosted at the Roswell Park Comprehensive Cancer 
Center. ${ }^{13}$ The subjective impact of salivary gland dysfunction and xerostomia was assessed by using the University of Michigan 15-item Xerostomia-related Quality of Life Scale (XeQoLS), which was self-administered by using a webbased version of the survey that was developed with REDCap. The XeQoLS has been previously validated in a comparable HNC cohort and contains 15 items that are specific to xerostomia covering 4 major domains of oral health-related quality of life, including physical (4 items), personal/psychological (4 items), social functioning (3 items), and pain/discomfort issues (4 items), creating a final score between 0 and 60 , with higher scores indicating worse symptoms. ${ }^{13}$ Surveys were administered at baseline (within 4 week before first ALTENS), and at 6, 9, 15, and 21 months from the start of ALTENS treatment.

\section{Toxicity assessment}

Acute adverse events were assessed according to the National Cancer Institute (NCI) Common Terminology Criteria for Adverse Events version 4.0 (CTCAE Version 4.0) at each treatment visit and follow-up assessment.

\section{Statistical methods}

The primary study objective was to determine whether ALTENS treatment using a four-times weekly schedule for 6 weeks is superior for reducing overall xerostomia burden, using the University of Michigan 15-item XeQoLS, compared with the standard ALTENS twice-weekly schedule for 12 weeks, at 15 months from the start of ALTENS treatment. A 15-month endpoint was used to capture a sustained response between the two groups. Secondary objectives were to: (1) study the primary endpoint at different time points $(6,9$, and 21 months) from the start of ALTENS treatment; (2) study the effect of ALTENS therapy on the
EORTC QOL measures at 6, 9, 15, and 21 months; and (3) compare the toxicities of both regimens.

A sample size of 15 patients per arm would yield $90 \%$ power to detect an effect size of 1.2 based on a two-sided test with an alpha of 0.05 and would produce a $95 \%$ confidence interval for between-treatment difference with a half-width of 0.748 times the within-group standard deviation. Differences in demographics of the treatment arms were assessed by using Pearson chi-square test for categorical variables and Wilcoxon Rank-Sum test for continuous variables. All patients with at least one post-baseline assessment for the primary outcome were considered evaluable for efficacy. Scatter plots were used to visualize the change in the overall XeQoLS score over time. A randomeffects generalized linear model was used to model the overall XeQoLS score adjusted for a random time effect, a fixed treatment arm, and the interaction of time and treatment. A 0.05 nominal significance level was used in all hypothesis testing. All statistical analyses were completed by using SAS version 9.4, statistical software (SAS Institute, Inc., Cary, NC).

\section{Results}

A total of 30 patients were randomized: 15 patients in the ALTENS twice weekly for the 12 weeks group and 15 patients in the ALTENS four times weekly for the 6 weeks group. Arms were balanced for age, gender, race, stage, histology, primary subtype, type of treatment and time from radiation to ALTENS treatment (Table 1), as well as baseline XeQoLS scores $(p=0.81$, Table 2$)$. Median patient age was 61 years (range, 46 to 78 years), and 26 patients were men $(87 \%)$. All patients in both arms completed all sessions. The XeQoLS significantly decreased for all patients (although not statistically in each arm) from a mean of 22 and 21 at baseline (in the four times per week and twice

Table 1. Descriptive Characteristics for Overall Cohort

\begin{tabular}{|c|c|c|c|c|c|}
\hline & & A: 4 times per week & B: 2 times per week & Overall & $\mathrm{p}$ \\
\hline Overall count & $n$ & 15 & 15 & 30 & \\
\hline \multirow[t]{2}{*}{ Age } & Mean/standard deviation & $61.1 / 7.5$ & $62.6 / 8.9$ & $61.9 / 8.1$ & 0.53 \\
\hline & Median/minimum/maximum & $59.1 / 52.2 / 75.3$ & 61.4/46.4/78.8 & $61.0 / 46.4 / 78.8$ & \\
\hline \multirow[t]{2}{*}{ Gender } & Male & $13(87 \%)$ & $13(87 \%)$ & $26(87 \%)$ & 1.0 \\
\hline & Female & $2(13 \%)$ & $2(13 \%)$ & $4(13 \%)$ & \\
\hline \multirow[t]{2}{*}{ Race } & White & $15(100 \%)$ & $14(93 \%)$ & $29(97 \%)$ & 0.31 \\
\hline & Asian & & $1(7 \%)$ & $1(3 \%)$ & \\
\hline \multirow[t]{4}{*}{ Stage } & I & 0 & $1(7 \%)$ & $1(3 \%)$ & 0.27 \\
\hline & II & $1(7 \%)$ & $4(27 \%)$ & $5(17 \%)$ & \\
\hline & III & $3(20 \%)$ & $3(20 \%)$ & $6(20 \%)$ & \\
\hline & IV & $11(73 \%)$ & $7(47 \%)$ & $18(60 \%)$ & \\
\hline \multirow[t]{2}{*}{ Histology } & Squamous cell Ca. & $12(80 \%)$ & $12(80 \%)$ & $24(80 \%)$ & 1.0 \\
\hline & Other & $3(20 \%)$ & $3(20 \%)$ & $6(20 \%)$ & \\
\hline \multirow[t]{6}{*}{ Primary } & Oral cavity & $5(33 \%)$ & $3(20 \%)$ & $8(27 \%)$ & 0.36 \\
\hline & Oropharynx & $3(20 \%)$ & $7(47 \%)$ & $10(33 \%)$ & \\
\hline & Larynx & $2(13 \%)$ & $3(20 \%)$ & $5(17 \%)$ & \\
\hline & Hypopharynx & $1(7 \%)$ & 0 & $1(3 \%)$ & \\
\hline & Nasopharynx & $2(13 \%)$ & $2(13 \%)$ & $4(13 \%)$ & \\
\hline & Unknown primary & $2(13 \%)$ & 0 & $2(7 \%)$ & \\
\hline \multirow[t]{2}{*}{ Treatment } & Radiation alone & 0 & $2(20 \%)$ & $2(7 \%)$ & 0.14 \\
\hline & Chemoradiation & $15(100 \%)$ & $13(80 \%)$ & $28(93 \%)$ & \\
\hline \multirow[t]{2}{*}{ Time from RT } & $<6$ months & $8(53 \%)$ & $7(47 \%)$ & $15(50 \%)$ & 0.72 \\
\hline & $>6-12$ months & $7(47 \%)$ & $8(53 \%)$ & $15(50 \%)$ & \\
\hline
\end{tabular}


Table 2. Overall XeQOL Scores at Each Visit for Patients Receiving Altens 4 Times Per Week for 6 Weeks (Arm A) and Patients Receiving Altens Twice Weekly for 12 Weeks (Arm B)

\begin{tabular}{|c|c|c|c|c|c|c|c|c|c|c|c|}
\hline \multirow[b]{2}{*}{$\begin{array}{l}\text { Overall XeQOL } \\
\text { score }\end{array}$} & \multicolumn{10}{|c|}{ Visit } & \multirow[b]{2}{*}{ Total } \\
\hline & Baseline & 2 week & 6 week & 8 week & 10 week & 12 week & 6 month & 9 month & $\begin{array}{c}15 \\
\text { month }\end{array}$ & $\begin{array}{c}21 \\
\text { month }\end{array}$ & \\
\hline \multicolumn{12}{|l|}{ A: 4 times per week } \\
\hline$n$ & 15 & 1 & - & - & 2 & 14 & 12 & 6 & 8 & 4 & \\
\hline Mean & 15.21 & 39 & - & - & 32 & 14.86 & 13.92 & 10.5 & 12.25 & 10.5 & \\
\hline Standard deviation & 17.09 & - & - & - & 12.73 & 12.23 & 15.7 & 15.12 & 14.31 & 18.38 & \\
\hline \multicolumn{12}{|l|}{ B: 2 times per week } \\
\hline$n$ & 15 & 2 & 10 & 2 & 1 & 5 & 9 & 6 & 7 & 5 & \\
\hline Mean & 17.89 & 30 & 15.5 & 20.5 & 58 & 13 & 14.56 & 14 & 12 & 5.8 & \\
\hline Standard deviation & 18.22 & 5.66 & 10.83 & 9.19 & . & 17.28 & 17.31 & 13.05 & 20.05 & 4.97 & \\
\hline$p$-value ${ }^{a}$ & & 0.04 & 0.22 & 0.22 & 0.001 & 0.42 & 0.27 & 0.34 & 0.46 & $*$ & 0.007 \\
\hline p-value & 0.81 & 0.83 & $*$ & $*$ & 0.13 & 0.81 & 0.64 & 0.52 & 0.71 & $*$ & 0.68 \\
\hline
\end{tabular}

${ }^{\mathrm{a}}$ Comparison over time for all patients.

${ }^{\mathrm{b}}$ Comparison between arms.

*Unable to calculate.

ALTENS, acupuncture-like transcutaneous electrical nerve stimulation.

weekly arms, respectively) to 12 in both arms at 15 months ( $p=0.007$, Fig. 1 and Table 2). There was no difference between arms $(p=0.68$, Table 2$)$.

For the four times per week group, XeQoLS was 14 at 6 months, 11 at 9 months, 12 at 15 months, and 11 at 21 months. For the twice-weekly arm, XeQoLS was 15 at 6 months, 14 at 9 months, 12 at 15 months, and 6 at 21 months. There were only three total reported adverse events (a grade 1 generalized paresthesia, a grade 2 low back ache, and a grade 3 abdominal cramp). All of these were self-limited, not temporally associated with treatment, and none was attributable to the ALTENS.

\section{Discussion}

This randomized study of response to ALTENS treatment, four times weekly for 6 weeks versus twice weekly for 12 weeks, showed that XeQoLS decreased from a mean of 22 and 21 at baseline (in the four times per week and twice weekly arms) to 12 in both arms at 15 months. This suggests that effective and sustained treatment responses were achieved in both arms of the study. In addition, both treatment regimens were well tolerated with no attributable adverse events. Although our primary endpoint of superior efficacy was negative, these results are the first to demonstrate that a four-times weekly ALTENS regimen for 6 weeks is as equally effective as the conventional schedule of twice weekly for 12 weeks. Although the shorter time frame did not appear to improve treatment efficacy, this shorter regimen may appeal to patients who seek to finish ALTENS treatment in a quicker time frame and improve compliance.
FIG. 1. Overall XeQOL score at each visit for patients receiving ALTENS four times per week for 6 weeks ( $\operatorname{arm} \mathrm{A})$ and patients receiving ALTENS twice weekly for 12 weeks (arm B). ALTENS, acupuncture-like transcutaneous electrical nerve stimulation.

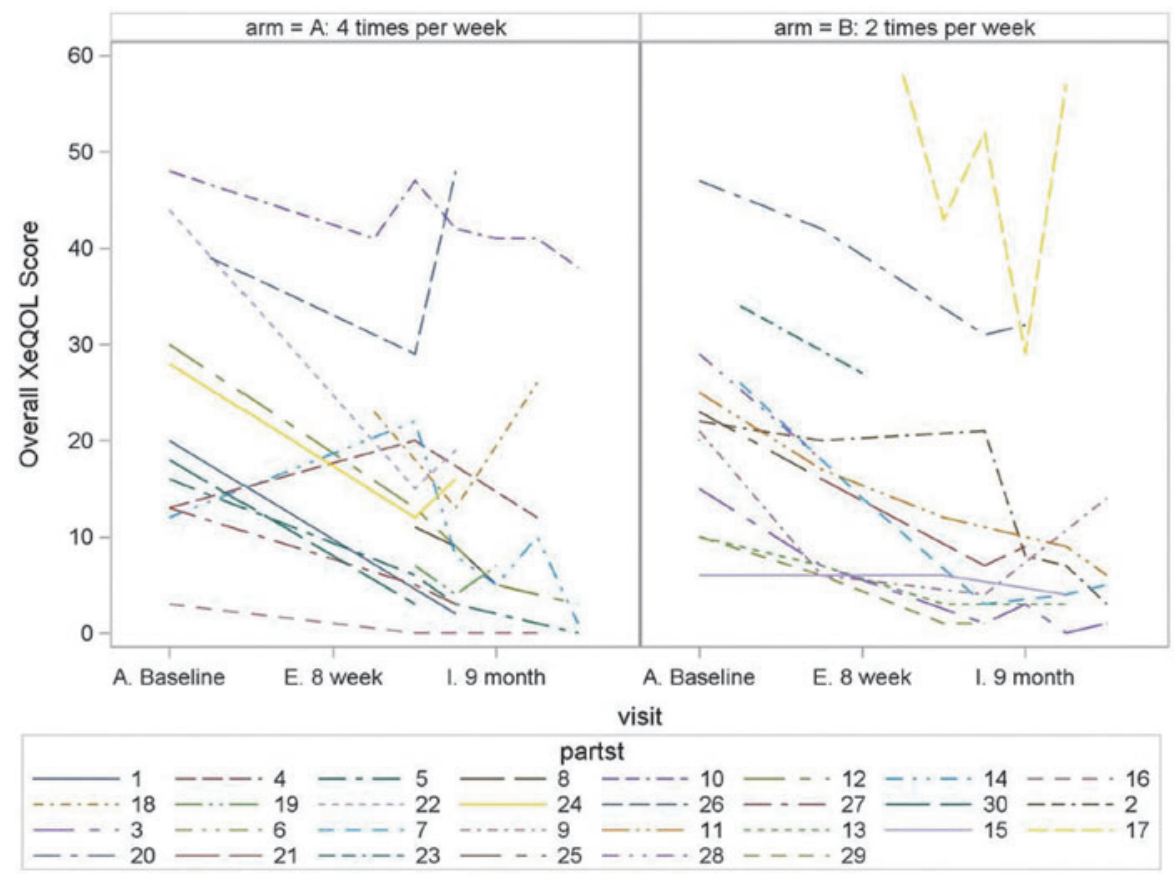


The xerostomia reduction observed in the group receiving ALTENS twice weekly for 12 weeks is consistent with previously published reports. ${ }^{7-10}$ Two randomized studies have evaluated the efficacy of ALTENS. In a phase I-II randomized trial, Wong et al. evaluated twice weekly for 12 weeks (with a 2-week break after 6 weeks) ALTENS for radiation-induced xerostomia in HNC patients. ${ }^{9}$ The authors found a significant improvement in xerostomia scores at all time points after ALTENS, with no toxicity reported. RTOG 0537 evaluated the feasibility and efficacy of ALTENS in a phase II/III randomized trial. The phase II results indicated the feasibility of twice weekly for 12 weeks ALTENS with a 94\% compliance rate; the phase III results showed no significant difference in overall xerostomia burden between patients randomized to oral pilocarpine compared with 12 weeks of ALTENS. $^{7,8}$ However, toxicity was significantly greater in the pilocarpine group. ${ }^{7}$ In our experience, the majority of patients simply refuse or fail to comply with pilocarpine, thus the use of ALTENS is often preferred. Treatment with ALTENS over a shorter time frame may make this option even more appealing.

This study is also consistent with previous randomized trials evaluating traditional acupuncture versus usual oral care for treatment of xerostomia. ${ }^{14,15}$ Simcock et al. demonstrated eight sessions of weekly group acupuncture compared with group oral care education provided it significantly improved symptomatic relief in patients suffering from chronic radiation-induced xerostomia. ${ }^{14}$ In another study, Pfister et al. found a significant reduction in xerostomia in patients receiving acupuncture versus usual oral care. ${ }^{15}$ Similar to our trial, these studies based their results on improvements in patient-reported outcomes. ALTENS offers a less-invasive alternative to traditional acupuncture that may be more accessible to patients.

There could be a few explanations as to why the 6-week ALTENS regimen was not superior to the 12-week regimen as hypothesized via increased neural action. One reason could be that the shortened regimen was not accelerated enough to see an observable difference. Perhaps a more frequent regimen with daily ALTENS or longer treatment sessions are required to see a larger effect. Another cause could be that the ability of ALTENS to improve xerostomia symptoms has already been maxed out on the existing regimen. Further studies are warranted to examine other ways to maximize the effect of ALTENS on reducing xerostomia burden.

There are several strengths and limitations to this trial. This study was able to directly compare two ALTENS regimens in randomized cohorts with similar clinical characteristics to examine whether an accelerated ALTENS course was superior in improving xerostomia outcomes. Compliance was $100 \%$ in both cohorts, and ALTENS was performed in a standardized manner for each patient at a single institution. Despite these advantages, this study was limited by the size of the patient cohort and future trials would benefit from a larger cohort. Although XeQoLS is a validated tool to subjectively evaluate xerostomia in HNC patients, another limitation is lack of objective data such as whole salivary production to further verify our results. In addition, there was loss to follow-up that may limit our interpretation of the results; however, based on the observed sample size at 15 months, the generalized linear model has $90 \%$ power to detect differences of at least 1.82 standard deviations.

\section{Future directions}

Recognizing that the Codetron TENS unit used in all prospective studies (including this one) is no longer in production and that time in clinical practices is limited, our group has described successfully treating a patient with xerostomia by using a protocol for patients to selfadminister ALTENS treatment at home, with a commercially available and cheap device (roughly $\$ 300){ }^{16}$ To further improve the convenience of treatment for these patients, our group would like to further evaluate the efficacy of unmonitored home use of ALTENS by initiating a multiinstitutional study enabling other treatment centers to loan ALTENS units to HNC survivors with xerostomia.

\section{Conclusions}

This study demonstrates that both ALTENS regimens appear to be safe, well tolerated, and equally effective. We now routinely make ALTENS units available for home use. The next step will be to see whether larger groups of HNC patients will have similar benefits with unmonitored home use.

\section{Acknowledgments}

The authors are grateful to Raimond KW Wong for his pioneering work in the field and ongoing collaboration with their program; Debbie Neimanis for her tireless dedication as a research nurse, which made analysis of this study possible.

\section{Author Disclosure Statement}

No competing financial interests exist.

\section{Funding Information}

This work was supported by Roswell Park Comprehensive Cancer Center and National Cancer Institute (NCI) grant P30CA016056 and the Roswell Park Alliance Foundation.

\section{References}

1. Fung-Kee-Fung SD, Hackett R, Hales L, et al. A prospective trial of volumetric intensity-modulated arc therapy vs conventional intensity modulated radiation therapy in advanced head and neck cancer. World J Clin Oncol 2012;3: 57-62.

2. Franzen L, Funegard U, Ericson T, Henriksson R. Parotid gland function during and following radiotherapy of malignancies in the head and neck. A consecutive study of salivary flow and patient discomfort. Eur J Cancer 1992;28: 457-462.

3. Greenspan D. Xerostomia: Diagnosis and management. Oncology (Williston Park) 1996;10(3 Suppl):7-11.

4. Chambers MS, Rosenthal DI, Weber RS. Radiationinduced xerostomia. Head Neck 2007;29:58-63.

5. Scarantino C, LeVeque F, Swann RS, et al. Effect of pilocarpine during radiation therapy: Results of RTOG 97-09, a phase III randomized study in head and neck cancer patients. J Support Oncol 2006;4:252-258. 
6. Warde P, O'Sullivan B, Aslanidis J, et al. A Phase III placebo-controlled trial of oral pilocarpine in patients undergoing radiotherapy for head-and-neck cancer. Int $\mathrm{J}$ Radiat Oncol Biol Phys 2002;54:9-13.

7. Wong RK, Deshmukh S, Wyatt G, et al. Acupuncture-like transcutaneous electrical nerve stimulation versus pilocarpine in treating radiation-induced xerostomia: Results of RTOG 0537 Phase 3 Study. Int J Radiat Oncol Biol Phys 2015;92:220-227.

8. Wong RK, James JL, Sagar S, et al. Phase 2 results from Radiation Therapy Oncology Group Study 0537: A phase 2/3 study comparing acupuncture-like transcutaneous electrical nerve stimulation versus pilocarpine in treating early radiation-induced xerostomia. Cancer 2012;118:4244-4252.

9. Wong RK, Jones GW, Sagar SM, et al. A Phase I-II study in the use of acupuncture-like transcutaneous nerve stimulation in the treatment of radiation-induced xerostomia in head-and-neck cancer patients treated with radical radiotherapy. Int J Radiat Oncol Biol Phys 2003;57:472-480.

10. Wyatt G, Pugh SL, Wong RK, et al. Xerostomia healthrelated quality of life: NRG oncology RTOG 0537. Qual Life Res 2016;25:2323-2333.

11. Haker E, Egekvist H, Bjerring P. Effect of sensory stimulation (acupuncture) on sympathetic and parasympathetic activities in healthy subjects. J Auton Nerv Syst 2000;79: 52-59.

12. Platek ME, McCloskey SA, Cruz M, et al. Quantification of the effect of treatment duration on local-regional failure after definitive concurrent chemotherapy and intensitymodulated radiation therapy for squamous cell carcinoma of the head and neck. Head Neck 2013;35:684-688.

13. Henson BS, Inglehart MR, Eisbruch A, Ship JA. Preserved salivary output and xerostomia-related quality of life in head and neck cancer patients receiving parotid-sparing radiotherapy. Oral Oncol 2001;37:84-93.

14. Simcock R, Fallowfield L, Monson K, et al. ARIX: a randomised trial of accupuncture $\mathrm{v}$ oral care sessions in patients with chronic xerostomia following treatment of head and neck cancer. Ann Oncol 2013;24:776-783.

15. Pfister DG, Cassileth BR, Deng GE, et al. Accupuncture for pain and dysfunction after neck dessection: results of a randomized controlled trial. J Clin Oncol 2010;28:25652570.

16. Iovoli AJ, Singh AK. Accupuncture-like transcutaneous electrical nerve stimulation therapy success using a commercially available unit 8 years post-radiation for xerostomia: A case report. J Radiother Pract 2017;16:217-220.

Address correspondence to: Anurag K. Singh, MD Department of Radiation Medicine Roswell Park Comprehensive Cancer Center 665 Elm St Buffalo, NY 14203

E-mail: anurag.singh@roswellpark.org 